
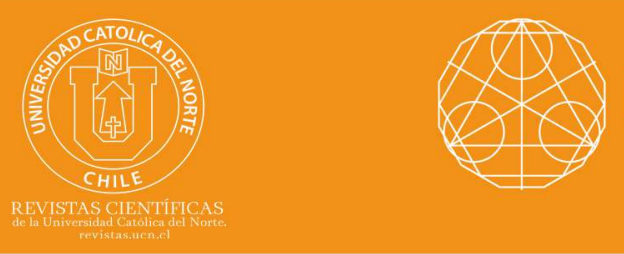

\title{
Bipolar quadripartitioned single valued neutrosophic sets
}

Kalyan Sinha1 ${ }^{1}$ orcid.org/0000-0002-8491-6168

Pinaki Majumdar² (10 orcid.org/0000-0001-9997-702X

${ }^{1}$ Acharya Brojendra Nath Seal College, Dept. of Mathematics, Koch Bihar, WB, India.

kalyansinha90@gmail.com

${ }^{2}$ Maharajadhiraj Uday Chand Women's College, Dept. of Mathematics, Burdwan, WB, India.

־pmajumdar2@rediffmail.com

\section{Abstract:}

The notion of simple bipolar quadripartition is presented valuable neutrosophic set. Some basic set theoretic terminologies, operations and properties of bipolar quadripartitioned single valued neutrosophic set are given here. Also different types of distances, similarity measures and entropy measure are discussed. Finally a decision making problem using the similarity measure technique of bipolar quadripartitioned single valued neutrosophic sets has been solved.

Keywords: Single valued neutrosophic set; Quadripartitioned single valued neutrosophic sets; Bipolar quadripartitioned single valued neutrosophic set.

MSC (2020): 03E20, 03E75.

\section{Cite this article as (IEEE citation style):}

K. Sinha and P. Majumdar, "Bipolar quadripartitioned single valued neutrosophic sets", Proyecciones (Antofagasta, On line),

vol. 39, no. 6, pp. 1597-1614, Dec. 2020, doi:

10.22199/issn.0717-6279-2020-06-0095.

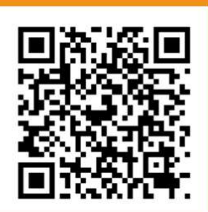

Article copyright: (C) 2020 Kalyan Sinha and Pinaki Majumdar. This is an open access article distributed under the terms of the Creative Commons License, which permits unrestricted use and distribution provided the original author and source are credited. 


\section{Introduction}

To deal with uncertainty based real and scientific problems, Prof. Zadeh [24] introduced the fuzzy set as a constructive tool. Later on Prof. Atanassov [1] extended the concept of fuzzy set theory to the intuitionstic fuzzy set (IFS), in which every element has both a membership degree and a nonmembership degree. It is quite clear that IFS are more helpful than fuzzy set theory to deal the various types of uncertainty model. In 2005, Smarandache [16] introduced the concept of a neutrosophic set (NS) as a further generalization of IFS from philosophical point of view. Gradually neutrosophic sets become more powerful technique to represent incomplete, inconsistent and indeterminate information which exists in our real world. In neutrosophic set, truth membership functions $\left(T_{A}\right)$, indeterminacy membership functions $\left(I_{A}\right)$, and falsity membership functions $\left(F_{A}\right)$ are represented independently. But in case of NS, all components lie in $] 0^{-}, 1^{+}[$. Thus it is very tough to apply NS sets in real life problems. To solve this problem Wang et al. [19] introduced single valued NS sets in 2010.

Recently bipolar fuzzy set and set theoretic operations based on fuzzy bipolar sets are introduced by Lee et al. in their paper $[8,9]$. They have shown that a bipolar fuzzy set consists two independent parts, positive membership degree $T^{+} \rightarrow[0,1]$ and a negative membership degree $T^{-} \rightarrow[-1,0]$. Later on several researchers have studied bipolar fuzzy sets and applied it to different socio-economic model [14, 15, 20, 21, 22, 23]. As a continuation of neutrosophic set, Deli et al. [6] introduced the concept of bipolar neutrosophic sets, where each element has both +ve and -ve neutrosophic degrees. Here, the positive membership degree $T_{A}^{+}, I_{A}^{+}, F_{A}^{+}$ denotes the truth membership, indeterminate membership and false membership of an element $x \in X$ corresponding to a bipolar neutrosophic set $A$ and the negative membership degree $T_{A}^{-}, I_{A}^{-}, F_{A}^{-}$denotes the truth membership, indeterminate membership and false membership of an element $x \in X$ to some anti-property corresponding to a bipolar neutrosophic set $A$.

On the other hand, another extension of single valued neutrosophic sets, i.e. quadripartitioned single valued neutrosophic set was introduced by R. Chatterjee et al. [5] in 2016. It is four valued logic set $A$ consisting truthmembership $T_{A}$, a contradiction membership $C_{A}$, an ignorance-membership $U_{A}$ and a falsity membership $F_{A}$ for each $x \in X$. Now quadripartitioned single valued neutrosophic set becomes an important tool in solving various types of decision making problems, medical diagnosis problems, clustering 
problems etc. In this paper we have introduced the bipolar quadripartitioned single valued neutrosophic set (BQSVNS) for the first time. Here we use BQSVNS to represent bipolar quadripartitioned single valued neutrosophic set. The organization of this paper have been done as follows: In Section 2, preliminary concepts regarding the paper have been discussed with proper citations. We have defined BQSVNS and it's various types of operations in Section 3. Section 4 gives us an idea of different type of similarity measures of BQSVNS. In Section 5, we have tried to solve a real life problem using BQSVNS. Section 6 concludes the paper.

\section{Preliminaries}

Prof. Smarandache introduced the term 'neutrosophy' as a branch of philosophy. Neutrosophic sets play an important role in solving many real life problems. There are many types of neutrosophic sets and each of these types have several applications $[2,4,10,17,19]$.

Definition 2.1. [16] Suppose $U$ be a universe. A neutrosophic sets (NS) $A$ in $U$ is characterized by a truth-membership function $T_{A}$, an indeterminacy membership function $I_{A}$ and a falsity-membership function $F_{A}$. Here for each $x \in U, T_{A}(x), I_{A}(x)$ and $F_{A}(x)$ are real non-standard elements of ] $0^{-}, 1^{+}[$. A can be written as:

$A=\left\{\left\langle\left(x, T_{A}(x), I_{A}(x), F_{A}(x)\right): x \in U, T_{A}(x), I_{A}(x), F_{A}(x) \in\right] 0^{-}, 1^{+}[\rangle\right\}$.

There is no restriction on the sum of $T_{A}(x), I_{A}(x), F_{A}(x)$, i.e,

$$
0^{-} \leq T_{A}(x), I_{A}(x), F_{A}(x) \leq 3^{+} .
$$

But the definition of neutrosophic sets creates many difficulties to apply in real life problems. Thus the idea of single valued neutrosophic set, which is an generalization of a neutrosophic set is introduced.

Definition 2.2. [19] Let $U$ be a universe. A single valued neutrosophic set (SVNS) $A$ in $U$ is characterized by a truth-membership function $T_{A}$, an indeterminacy membership function $I_{A}$ and a falsity-membership function $F_{A} \cdot T_{A}(x), I_{A}(x)$ and $F_{A}(x)$ are real standard elements of $[0,1]$. It can be written as

$$
A=\left\{\left\langle\left(x, T_{A}(x), I_{A}(x), F_{A}(x)\right): x \in U, T_{A}(x), I_{A}(x), F_{A}(x) \in[0,1]\right\rangle\right\} .
$$


Gradually research develops based on the single valued neutrosophic set theory. In 2016, R. Chatterjee et al. introduced the quadripartitioned neutrosophic set and studied its properties in their paper [5].

Definition 2.3. Suppose $X$ be a non-empty set. A quadripartitioned neutrosophic set (QSVNS) $A$ over $X$ characterizes each element $x$ in $X$ by a truth-membership function $T_{A}$, a contradiction membership function $C_{A}$, an ignorance-membership function $U_{A}$ and a falsity membership function $F_{A}$ such that for each $x \in X, T_{A}(x), C_{A}(x), U_{A}(x), F_{A}(x) \in[0,1]$ and $0 \leq T_{A}(x)+C_{A}(x)+U_{A}(x)+F_{A}(x) \leq 4$.

On the other hand, Prof I. Deli et al. introduced the bipolar neutrosophic sets in 2015 and studied the properties of bipolar neutrosophic sets in their paper [6]. They defined the bipolar neutrosophic sets as follows:

Definition 2.4. A bipolar neutrosophic set $A$ over a non-empty set $X$ is defined as an object of the form

$$
A=\left\{\left\langle\left(x, T_{A}^{+}(x), I_{A}^{+}(x), F_{A}^{+}(x), T_{A}^{-}(x), I_{A}^{-}(x), F_{A}^{-}(x)\right): x \in X\right\rangle\right\} .
$$

where $T_{A}^{+}(x), I_{A}^{+}(x), F_{A}^{+}(x): X \rightarrow[0,1]$ and $T_{A}^{-}(x), I_{A}^{-}(x), F_{A}^{-}(x): X \rightarrow$ $[-1,0]$.

The set theoretic properties and many other properties i.e. entropy, various types of similarity measures of a neutrosophic sets, single valued neutrosophic sets, bipolar neutrosophic sets etc can be found in any of the monograph say $[16,17,18]$. In this paper we will introduce the notion of bipolar quadripartitioned single valued neutrosophic set for the first time.

\section{Bipolar quadripartitioned single valued neutrosophic set}

In this section, we will introduce the concept of BQSVNS and define some algebraic operations on them.

Definition 3.1. Suppose $X$ be a non-empty set. A bipolar quadripartitioned single valued neutrosophic set (BQSVNS) A, over $X$ characterizes each element $x$ in $X$ by a positive truth-membership function $T_{A}^{+}$, a positive contradiction membership function $C_{A}^{+}$, a positive ignorance-membership function $U_{A}^{+}$, a positive falsity membership function $F_{A}^{+}$, a negative truthmembership function $T_{A}^{-}$, a negative contradiction membership function 
$C_{A}^{-}$, a negative ignorance-membership function $U_{A}^{-}$, a negative falsity membership function $F_{A}^{-}$, such that for each $x \in X, T_{A}^{+}, C_{A}^{+}, U_{A}^{+}, F_{A}^{+} \in[0,1]$, $T_{A}^{-}, C_{A}^{-}, U_{A}^{-}, F_{A}^{-} \in[-1,0]$ and

$$
0 \leq T_{A}^{+}+C_{A}^{+}+U_{A}^{+}+F_{A}^{+} \leq 4,-4 \leq T_{A}^{-}+C_{A}^{-}+U_{A}^{-}+F_{A}^{-} \leq 0
$$

When $X$ is discrete, $A$ is represented as

$$
A=\sum_{i=1}^{n}\left\langle T_{A}^{+}, C_{A}^{+}, U_{A}^{+}, F_{A}^{+}, T_{A}^{-}, C_{A}^{-}, U_{A}^{-}, F_{A}^{-}\right\rangle / x_{i}, x_{i} \in X .
$$

On the other hand, for continuous universe $X$, we can write $A$ as

$$
A=\int\left\langle T_{A}^{+}, C_{A}^{+}, U_{A}^{+}, F_{A}^{+}, T_{A}^{-}, C_{A}^{-}, U_{A}^{-}, F_{A}^{-}\right\rangle / x, x \in X .
$$

Example 3.2. Consider the case where four meteorologists $x_{1}, x_{2}, x_{3}, x_{4}$ were asked to give their opinion on the statement "There will be normal monsoon in India" in the year 2020. Each of the four meteorologists will give their opinion in terms of degree of agreement, agreement or disagreement both, neither agreement nor disagreement, disagreement in terms of degree of positive and negative arguments respectively. The aggregate of their opinion is very well expressed by a BQSVNS A as follows:

$$
\begin{aligned}
A= & \langle 0.8,0.6,0.2,0.2,-0.7,-0.6,-0.3,-0.1\rangle / x_{1}+ \\
& \langle 0.6,0.7,0.4,0.1,-0.6,-0.8,-0.3,-0.2\rangle / x_{2}+ \\
& \langle 0.5,0.5,0.7,0.1,-0.5,-0.6,-0.8,-0.2\rangle / x_{3}+ \\
& \langle 0.4,0.6,0.6,0.8,-0.5,-0.5,-0.6,-0.8\rangle / x_{4} .
\end{aligned}
$$

Here according to $x_{1}$, the degree of agreement with the statement is 0.8 and the degree of negative agreement with the statement is 0.7 , the degree of both agreement and disagreement is 0.6 and the degree of negative argument of "both agreement and disagreement" is 0.6. The degree of neither agreement nor disagreement is 0.2 , while the degree of negative argument of it is 0.3. Similarly the degree of disagreement with the statement is 0.2 and degree of negative disagreement is 0.1. This is how BQSVNS set has been made.

Definition 3.3. A BQSVNS $B$ over $X$ is said to be an strongly absolute $B Q S V N S$, denoted by $B^{s}$, if and only if its membership values are respectively defined as $T_{B}^{+}(x)=1, C_{B}^{+}(x)=1, U_{B}^{+}(x)=0, F_{B}^{+}(x)=0, T_{B}^{-}(x)=$ $0, C_{B}^{-}(x)=0, U_{B}^{-}(x)=0, F_{B}^{-}(x)=0, \forall x \in X$. 
Definition 3.4. A BQSVNS $B$ over $X$ is said to be an weekly absolute $B Q S V N S$, denoted by $B^{w}$, if and only if its membership values are respectively defined as $T_{B}^{+}(x)=0, C_{B}^{+}(x)=0, U_{B}^{+}(x)=0, F_{B}^{+}(x)=0, T_{B}^{-}(x)=$ $0, C_{B}^{-}(x)=0, U_{B}^{-}(x)=-1, F_{B}^{-}(x)=-1 \forall x \in X$.

Definition 3.5. A BQSVNS $B$ over $X$ is said to be an strongly null $B Q S V N S$, denoted by $\Phi^{s}$, if and only if its membership values are respectively defined as $T_{B}^{+}(x)=0, C_{B}^{+}(x)=0, U_{B}^{+}(x)=1, F_{B}^{+}(x)=1, T_{B}^{-}(x)=$ $0, C_{B}^{-}(x)=0, U_{B}^{-}(x)=0, F_{B}^{-}(x)=0 \forall x \in X$.

Definition 3.6. A BQSVNS B over $X$ is said to be an weekly null BQSVNS, denoted by $\Phi^{w}$, if and only if its membership values are respectively defined as $T_{B}^{+}(x)=0, C_{B}^{+}(x)=0, U_{B}^{+}(x)=0, F_{B}^{+}(x)=0, T_{B}^{-}(x)=-1, C_{B}^{-}(x)=$ $-1, U_{B}^{-}(x)=0, F_{B}^{-}(x)=0 \forall x \in X$.

Remark 3.7. A BQSVNS is a generalization of a bipolar neutrosophic set. If we take average the components $C^{+}, U^{+}$and $C^{-}, U^{-}$together respectively, we can easily get a bipolar SVN set.

Now we will introduce some set-theoretic operations on BQSVNS over a common universe $X$ and study some of their basic properties.

Definition 3.8. Suppose $A$ and $B$ are two BQSVNS over $X$. Then $A$ is said to be a subset of $B$ i.e. $A \subseteq B$ if and only if $T_{A}^{+}(x) \leq T_{B}^{+}(x), C_{A}^{+}(x) \leq$ $C_{B}^{+}(x), U_{A}^{+}(x) \geq U_{B}^{+}(x), F_{A}^{+}(x) \geq F_{B}^{+}(x), T_{A}^{-}(x) \geq T_{B}^{-}(x), C_{A}^{-}(x) \geq C_{B}^{-}(x), U_{A}^{-}(x) \leq$ $U_{B}^{-}(x), F_{A}^{-}(x) \leq F_{B}^{-}(x) \forall x \in X$.

Definition 3.9. Suppose $A=\sum_{i=1}^{n}\left\langle T_{A}^{+}, C_{A}^{+}, U_{A}^{+}, F_{A}^{+}, T_{A}^{-}, C_{A}^{-}, U_{A}^{-}, F_{A}^{-}\right\rangle / x_{i}, x_{i} \in$ $X$ is a BQSVNS over $X$. Then the complement of $A$, denoted by $A^{c}$ is as following:

$$
A^{c}=\sum_{i=1}^{n}\left\langle F_{A}^{+}, U_{A}^{+}, C_{A}^{+}, T_{A}^{+}, F_{A}^{-}, U_{A}^{-}, C_{A}^{-}, T_{A}^{-}\right\rangle / x_{i}, x_{i} \in X .
$$

Definition 3.10. Suppose $A$ and $B$ are two BQSVNS over $X$. Then the union of $A, B$ i.e. $A \cup B$ is defined as: $T_{A}^{+}(x) \vee T_{B}^{+}(x), C_{A}^{+}(x) \vee$ $C_{B}^{+}(x), U_{A}^{+}(x) \wedge U_{B}^{+}(x), F_{A}^{+}(x) \wedge F_{B}^{+}(x), T_{A}^{-}(x) \wedge T_{B}^{-}(x), C_{A}^{-}(x) \wedge C_{B}^{-}(x), U_{A}^{-}(x) \vee$ $U_{B}^{-}(x), F_{A}^{-}(x) \vee F_{B}^{-}(x) \forall x \in X$.

Definition 3.11. Suppose $A$ and $B$ are two BQSVNS over $X$. Then the intersection of $A, B$ i.e. $A \cap B$ is defined as: $T_{A}^{+}(x) \wedge T_{B}^{+}(x), C_{A}^{+}(x) \wedge$ $C_{B}^{+}(x), U_{A}^{+}(x) \vee U_{B}^{+}(x), F_{A}^{+}(x) \vee F_{B}^{+}(x), T_{A}^{-}(x) \vee T_{B}^{-}(x), C_{A}^{-}(x) \vee C_{B}^{-}(x), U_{A}^{-}(x) \wedge$ $U_{B}^{-}(x), F_{A}^{-}(x) \wedge F_{B}^{-}(x) \forall x \in X$. 
Example 3.12. Consider a BQSVNS B as follows:

$$
\begin{aligned}
B= & \langle 0.9,0.5,0.3,0.2,-0.5,-0.2,-0.1,-0.1\rangle / x_{1}+ \\
& \langle 0.7,0.8,0.3,0.2,-0.5,-0.7,-0.4,-0.1\rangle / x_{2}+ \\
& \langle 0.4,0.5,0.6,0.2,-0.6,-0.7,-0.9,-0.4\rangle / x_{3} \\
+ & \langle 0.4,0.5,0.7,0.8,-0.4,-0.6,-0.7,-0.8\rangle / x_{4} .
\end{aligned}
$$

It is assumed that the BQSVNS A in Example 3.2 and the above mentioned BQSVNS $B$ are defined over the same universal set $X$. Then we have the following:

$$
\begin{aligned}
A^{c}= & \langle 0.2,0.2,0.6,0.8,-0.1,-0.3,-0.6,-0.7\rangle / x_{1}+ \\
\langle & 0.1,0.4,0.7,0.6,-0.2,-0.3,-0.8,-0.6\rangle / x_{2}+ \\
& \langle 0.1,0.7,0.5,0.5,-0.2,-0.8,-0.6,-0.5\rangle / x_{3}+ \\
& \langle 0.8,0.6,0.6,0.4,-0.8,-0.6,-0.5,-0.5\rangle / x_{4} . \\
A \cup B= & \langle 0.9,0.6,0.2,0.2,-0.7,-0.6,-0.1,-0.1\rangle / x_{1}+ \\
& \langle 0.7,0.8,0.3,0.2,-0.6,-0.8,-0.3,-0.2\rangle / x_{2}+ \\
& \langle 0.5,0.5,0.6,0.1,-0.6,-0.7,-0.8,-0.2\rangle / x_{3}+ \\
& \langle 0.4,0.6,0.6,0.8,-0.5,-0.6,-0.6,-0.8\rangle / x_{4} . \\
& \\
A \cap B= & \langle 0.8,0.5,0.3,0.2,-0.5,-0.2,-0.3,-0.1\rangle / x_{1}+ \\
& \langle 0.6,0.7,0.4,0.2,-0.5,-0.7,-0.4,-0.2\rangle / x_{2}+ \\
& \langle 0.4,0.5,0.7,0.2,-0.5,-0.6,-0.8,-0.4\rangle / x_{3}+ \\
& \langle 0.4,0.5,0.7,0.8,-0.5,-0.6,-0.7,-0.8\rangle / x_{4} .
\end{aligned}
$$

Proposition 3.13. The set-theoretic axioms are satisfied by any BQSVNS as it can be easily verified. Consider BQSVNS sets $A, B, C$ over the same universe $X$. Then the following properties holds all for BQSVNS over $X$.

(i) $A \cup B=B \cup A ; A \cap B=B \cap A$.

(ii) $A \cup(B \cup C)=(A \cup B) \cup C ; A \cap(B \cap C)=(A \cap B) \cap C$ 
(iii) $A \cap(A \cup B)=A ; \quad A \cup(A \cap B)=A$.

(iv) $\left(A^{c}\right)^{c}=A$.

(v) $(A \cup B)^{c}=A^{c} \cap B^{c} ;(A \cap B)^{c}=A^{c} \cup B^{c}$

(vi) $A \cup A=A \cup A ; A \cap A=A \cap A$.

(vii) $A \cup \Phi^{s}=A ; A \cap \Phi^{s}=\Phi^{s}$.

(viii) $A \cup \Phi^{w}=A ; A \cap \Phi^{w}=\Phi^{w}$.

\section{BQSVNS and it's different similarity measures}

Consider the non-empty universe of discourse $X=\Phi$ and denote the set of BQSVNS over $X$ by $Q(X)$.

Definition 4.1. A mapping $s: Q(X) \times Q(X) \rightarrow[0,1]$ is said to be similarity measure iff for $A, B \in Q(X)$, the following properties are satisfied:

(S-i) $s(A, B)=s(B, A)$

(S-ii) $0 \leq s(A, B)<1$ and $s(A, B)=1$ iff $A=B$.

(S-iii) for any $A, B, C \in Q(X), A \subseteq B \subseteq C, s(A, C) \leq s(A, B) \wedge s(B, C)$.

It is seen that the condition $(S-i i i)$ in Definition 4.1 exists but many of the well known similarity measure techniques say weighted similarity measure, cosine similarity measures etc does not satisfy the above mentioned condition. Although the weighted similarity measure, cosine similarity measure etc techniques has huge application in solving different type of real life problems. So it is quite natural to introduce a definition of similarity measure omitting the condition $(S-i i i)$ of the Definition 4.1. In that case, we re-define the similarity measure as quasi-similarity measure between two BQSVNS over $X$.

Definition 4.2. Consider the non-empty universe of discourse $X=\Phi$ and denote the set of BQSVNS over $X$ by $Q(X)$. Then a mapping $s^{\prime}: Q(X) \times$ $Q(X) \rightarrow[0,1]$ is said to be quasi similarity measure iff for $A, B \in Q(X)$, the following properties are satisfied:

(S-i) $s(A, B)=s(B, A)$

(S-ii) $0 \leq s(A, B)<1$ and $s(A, B)=1$ iff $A=B$. 


\subsection{Distance based similarity measure}

Definition 4.3. A mapping $d: Q(X) \times Q(X) \rightarrow \mathbf{R}^{+}$, where $R^{+}$is the set of all positive real numbers, is said to be a distance measure for BQSVNS iff for $A, B, C \in Q(X)$, it satisfies the following properties:

$(\mathrm{D}-\mathrm{i}) d(A, B)=d(B, A)$

(D-ii) $d(A, B) \geq 0$ and $d(A, B)=0$ iff $A=B$.

(D-iii) $d(A, B) \leq d(A, C)+d(C, B)$.

Suppose $H, K \in Q(X)$. Then $d$ is a metric on $Q(X)$. Now we define the following distance measures:

Definition 4.4. The Hamming distance between $H$ and $K$ is defined as

$$
\begin{array}{r}
h(H, K)=\left\{\sum _ { j = 1 } ^ { n } \left(\left|T_{H}^{+}\left(x_{j}\right)-T_{K}^{+}\left(x_{j}\right)\right|+\left|C_{H}^{+}\left(x_{j}\right)-C_{K}^{+}\left(x_{j}\right)\right|+\right.\right. \\
\left|F_{H}^{+}\left(x_{j}\right)-F_{K}^{+}\left(x_{j}\right)\right|+\left|T_{H}^{-}\left(x_{j}\right)-T_{K}^{-}\left(x_{j}\right)\right| \\
+\left|C_{H}^{-}\left(x_{j}\right)-C_{K}^{-}\left(x_{j}\right)\right|+\left|F_{H}^{-}\left(x_{j}\right)-F_{K}^{-}\left(x_{j}\right)\right|+ \\
\left.\left.\left|U_{H}^{+}\left(x_{j}\right)-U_{K}^{+}\left(x_{j}\right)\right|+\left|U_{H}^{-}\left(x_{j}\right)-U_{K}^{-}\left(x_{j}\right)\right|\right)\right\}
\end{array}
$$

Definition 4.5. The Normalized Hamming distance between $H$ and $K$ is defined as $h_{N}(H, K)=\frac{1}{8 n}(h(H, K))$

Definition 4.6. The Euclidean distance $E(H, K)$ is defined as follows:

$$
\begin{array}{r}
E(H, K)=\left[\sum _ { j = 1 } ^ { n } \left(\left|T_{H}^{+}\left(x_{j}\right)-T_{K}^{+}\left(x_{j}\right)\right|^{2}+\left|C_{H}^{+}\left(x_{j}\right)-C_{K}^{+}\left(x_{j}\right)\right|^{2}+\right.\right. \\
\left|F_{H}^{+}\left(x_{j}\right)-F_{K}^{+}\left(x_{j}\right)\right|^{2}+\left|T_{H}^{-}\left(x_{j}\right)-T_{K}^{-}\left(x_{j}\right)\right|^{2}+ \\
\left|C_{H}^{-}\left(x_{j}\right)-C_{K}^{-}\left(x_{j}\right)\right|^{2}+\left|F_{H}^{-}\left(x_{j}\right)-F_{K}^{-}\left(x_{j}\right)\right|^{2}+ \\
\left.\left.\left|U_{H}^{+}\left(x_{j}\right)-U_{K}^{+}\left(x_{j}\right)\right|^{2}+\left|U_{H}^{-}\left(x_{j}\right)-U_{K}^{-}\left(x_{j}\right)\right|^{2}\right)\right]^{\frac{1}{2}} .
\end{array}
$$

Definition 4.7. The normalized Euclidean distance $Q(H, K)$ is defined as follows:

$$
Q(H, K)=\frac{1}{2 \sqrt{2 n}} E(H, K)
$$


Example 4.8. We have calculated different types of measures as defined above based on the two BQSVNS A in Example 3.2 and the BQSVNS B in Example 3.12. The results are as follows:

$$
\begin{array}{r}
h(A, B)=3.8, \\
h_{N}(A, B)=0.118, \\
E(A, B)=0.938, \\
Q(A, B)=0.166,
\end{array}
$$

It is seen from the recent papers of this area that distance measure between two sets becomes the most attractive research topic among the researchers. The distance based measure between the two sets has a huge practical applications. Several researchers have used different types of distance based measures to solve the uncertainties based real life problems which involves uncertainties [7, 10]. Also, different types of operators i.e. induced ordered weighted aggregation distance (IOWAD) operators, an extended version of the usual OWA operators are used to find out the distance measures in order to solve decision making problems $[11,12,13]$. However, we are only concerned to the study of distance-based similarity measures in this paper.

\subsection{Distance-Based Similarity Measure of BQSVNS}

We have defined several types of distances between a pair of BQSVNS $H$ and $K$ over the set $Q(X)$ in the previous section. Now using these distances we can also define similarity measures for BQSVNS. In the following, we now define a similarity measure based on Hamming Distance.

$$
S_{1}^{d}(H, K)=\frac{1}{1+h(H, K)} .
$$

Also we can define another similarity measure as:

$$
S_{2}^{d}(H, K)=e^{-\alpha \cdot h(H, K)},
$$

where $\alpha$ is a positive real number (parameter) called the steepness measure. Similarly using Euclidian distance, similarity measure can be defined as follows:

$$
S_{1}^{\prime}(H, K)=\frac{1}{1+E(H, K)} .
$$


Again we can define another similarity measure as

$$
S_{2}^{\prime}(H, K)=e^{-\alpha \cdot E(H, K)},
$$

where $\alpha$ is a positive real number (parameter) called the steepness measure.

Example 4.9. For BQSVNS $A$ in Example 3.2 and BQSVNS $B$ in Example 3.12, we have the following:

$$
S_{1}^{d}(A, B)=0.208, S_{1}^{\prime}(A, B)=0.516 .
$$

\subsection{Similarity measures of BQSVNS based on membership values}

Consider $H, K \in Q(X)$. For each $x_{i} \in X, i=1,2, \ldots, n$ and for $j=$ $1,2, \ldots, 8$ define the functions $h_{j}^{H, K}: X \rightarrow[0,1]$ respectively as

$$
\begin{array}{r}
h_{1}^{H, K}\left(x_{i}\right)=\left|T_{H}^{+}\left(x_{i}\right)-T_{K}^{+}\left(x_{i}\right)\right|, \\
h_{2}^{H, K}\left(x_{i}\right)=\left|F_{H}^{+}\left(x_{i}\right)-F_{K}^{+}\left(x_{i}\right)\right|, \\
h_{3}^{H, K}\left(x_{i}\right)=\frac{1}{3}\left(h_{1}^{H, K}\left(x_{i}\right)+h_{2}^{H, K}\left(x_{i}\right)+\left|C_{H}^{+}\left(x_{i}\right)-C_{K}^{+}\left(x_{i}\right)\right|\right), \\
h_{4}^{H, K}\left(x_{i}\right)=\left|U_{H}^{+}\left(x_{i}\right)-U_{K}^{+}\left(x_{i}\right)\right|, \\
h_{5}^{H, K}\left(x_{i}\right)=\left|T_{H}^{-}\left(x_{i}\right)-T_{K}^{-}\left(x_{i}\right)\right|, \\
h_{6}^{H, K}\left(x_{i}\right)=\left|F_{H}^{-}\left(x_{i}\right)-F_{K}^{-}\left(x_{i}\right)\right|, \\
h_{7}^{H, K}\left(x_{i}\right)=\frac{1}{3}\left(h_{1}^{H, K}\left(x_{i}\right)+h_{2}^{H, K}\left(x_{i}\right)+\left|C_{H}^{-}\left(x_{i}\right)-C_{K}^{-}\left(x_{i}\right)\right|\right), \\
h_{8}^{H, K}\left(x_{i}\right)=\left|U_{H}^{-}\left(x_{i}\right)-U_{K}^{-}\left(x_{i}\right)\right|
\end{array}
$$

The above defined functions measure the difference between the various membership values corresponding to the two sets $H$ and $K$ w.r.t. each $x_{i}$. Define a mapping,

$$
\widetilde{S}(H, K)=1-\frac{1}{8 n} \sum_{i=1}^{n} \sum_{j=1}^{8} h_{j}^{H, K}\left(x_{i}\right) .
$$

Now we have the following theorem:

Theorem 4.10. $\widetilde{S}(H, K)$ is a measure of similarity between the two BQSVNS $H, K \in Q(X)$. 


\section{Proof.}

It is clear from definition of BQSVNS that all the values of $T^{+}, C^{+}, U^{+}$, $F^{+}, T^{-}, C^{-}, U^{-}, F^{-}$for a BQSVNS $H$ or $K$ lie between $[-1,1]$. Among all these quantities, $T^{+}, C^{+}, U^{+}, F^{+}$has maximum value 1 and has the minimum value 0 . On the other hand, $T^{-}, C^{-}, U^{-}, F^{-}$has maximum value 0 and has the minimum value -1 . As a result all the values of $h_{j}^{H, K}\left(x_{i}\right)$, $\forall x_{i} \in X$ lies in 0 and 1 and hence

$$
0 \leq 1-\frac{1}{8 n} \sum_{i=1}^{n} \sum_{j=1}^{8} h_{j}^{H, K}\left(x_{i}\right) \leq 1 .
$$

Again $\widetilde{S}(H, K)=1$ implies that $\sum_{i=1}^{n} \sum_{j=1}^{8} h_{j}^{H, K}\left(x_{i}\right)=0 \forall x_{i} \in X$ i.e. $h_{j}^{H, K}\left(x_{i}\right)=0, \forall j=1, \ldots, 8$ which is only possible iff

$T_{H}^{+}\left(x_{i}\right)=T_{K}^{+}\left(x_{i}\right), C_{H}^{+}\left(x_{i}\right)=C_{K}^{+}\left(x_{i}\right), U_{H}^{+}\left(x_{i}\right)=U_{K}^{+}\left(x_{i}\right), F_{H}^{+}\left(x_{i}\right)=F_{K}^{+}\left(x_{i}\right)$,

$T_{H}^{-}\left(x_{i}\right)=T_{K}^{-}\left(x_{i}\right), C_{H}^{-}\left(x_{i}\right)=C_{K}^{-}\left(x_{i}\right), U_{H}^{-}\left(x_{i}\right)=U_{K}^{-}\left(x_{i}\right), F_{H}^{-}\left(x_{i}\right)=F_{K}^{-}\left(x_{i}\right)$,

$\forall x_{i} \in X$, i.e. $H=K$

Finally, we suppose that $H \subseteq K \subseteq J$. Now by the definition of BQSVNS,

$$
T_{H}^{+}\left(x_{i}\right) \leq T_{K}^{+}\left(x_{i}\right) \leq T_{J}^{+}\left(x_{i}\right), C_{H}^{+}\left(x_{i}\right) \leq C_{K}^{+}\left(x_{i}\right) \leq C_{J}^{+}\left(x_{i}\right), U_{H}^{+}\left(x_{i}\right) \geq U_{K}^{+}\left(x_{i}\right) \geq U_{J}^{+}\left(x_{i}\right),
$$

$F_{H}^{+}\left(x_{i}\right) \geq F_{K}^{+}\left(x_{i}\right) \geq F_{J}^{+}\left(x_{i}\right), T_{H}^{-}\left(x_{i}\right) \geq T_{K}^{-}\left(x_{i}\right) \geq T_{J}^{-}\left(x_{i}\right), C_{H}^{-}\left(x_{i}\right) \geq C_{K}^{-}\left(x_{i}\right) \geq C_{J}^{-}\left(x_{i}\right)$,

$U_{H}^{-}\left(x_{i}\right) \leq U_{K}^{-}\left(x_{i}\right) \leq U_{J}^{+}\left(x_{i}\right), F_{H}^{-}\left(x_{i}\right) \leq F_{K}^{-}\left(x_{i}\right) \leq F_{J}^{-}\left(x_{i}\right) \forall x_{i} \in X$.

Using the above relation, we have $\left|T_{H}^{+}\left(x_{i}\right)-T_{J}^{+}\left(x_{i}\right)\right|>\mid T_{K}^{+}\left(x_{i}\right)-$ $T_{J}^{+}\left(x_{i}\right) \mid, \forall x_{i} \in X$ and hence $h_{1}^{H, J}\left(x_{i}\right)>h_{1}^{K, J}$. In a similar reason, we can say $h_{j}^{H, J}\left(x_{i}\right)>h_{j}^{K, J}\left(x_{i}\right) \forall x_{i} \in X, j=1,2, \ldots, 8$. Thus, $\widetilde{S}(H, J)<\widetilde{S}(H, K) \wedge \widetilde{S}(K, J)$.

\subsection{Weighted Similarity Measure}

The weighted similarity measure between two BQSVNS $H, K \in Q(X)$ are defined as follows:

$$
S^{w}(H, K)=\left[1-\frac{1}{8 n} w_{i} \sum_{i=1}^{n} \sum_{j=1}^{8} h_{j}^{H, K}\left(x_{i}\right)\right]^{\frac{1}{k}},
$$

where $k$ is any integer defined to be the order of similarity, $w_{i}$ are the weights associated to the elements $x_{i}$ of the universe, $i=1,2, \ldots, n$ such that $\sum_{i=1}^{n} w_{i}=1$. Using the same proof procedure of the Theorem 4.10, one can easily see that $S^{w}(H, K)$ is also a measure of similarity between the two BQSVNS $H, K \in Q(X)$. 


\subsection{Correlation coefficient based Similarity Measure}

Definition 4.11. The correlation coefficient based similarity measure between two BQSVNS $H, K \in Q(X)$ can be defined as follows:

$$
S^{C}(H, K)=\frac{\left[\sum_{i=1}^{n} \frac{T_{H}^{+}\left(x_{i}\right) \cdot T_{K}^{+}\left(x_{i}\right)+C_{H}^{+}\left(x_{i}\right) \cdot C_{K}^{+}\left(x_{i}\right)+F_{H}^{+}\left(x_{i}\right) \cdot F_{K}^{+}\left(x_{i}\right)+U_{H}^{+}\left(x_{i}\right) \cdot U_{K}^{+}\left(x_{i}\right)+}{T_{H}^{-}\left(x_{i}\right) \cdot T_{K}^{-}\left(x_{i}\right)+C_{H}^{-}\left(x_{i}\right) \cdot C_{K}^{-}\left(x_{i}\right)+F_{H}^{-}\left(x_{i}\right) \cdot F_{K}^{-}\left(x_{i}\right)+U_{H}^{-}\left(x_{i}\right) \cdot U_{K}^{-}\left(x_{i}\right)}\right]}{\left[\sum_{i=1}^{n} \frac{\left(T_{H}^{+}\left(x_{i}\right)^{2}+C_{H}^{+}\left(x_{i}\right)^{2}+U_{H}^{+}\left(x_{i}\right)^{2}+F_{H}^{+}\left(x_{i}\right)^{2}\right)}{\left.\left(T_{H}^{-}\left(x_{i}\right)^{2}+C_{H}^{-}\left(x_{i}\right)^{2}+U_{H}^{-}\left(x_{i}\right)^{2}+F_{H}^{-}\left(x_{i}\right)\right)^{2}\right)}\right]},
$$

Remark 4.12. $S^{C}(H, K)$ is a quasi-similarity between $H, K$.

\subsection{Entropy Based Similarity Measure}

Definition 4.13. Suppose $X$ be a non-empty universe containing the elements $\left\{x_{i}: i=1, \ldots, n\right\}$. A mapping ent $: Q(X) \rightarrow[0,1]$ is said to be an entropy on BQSVNS if $E$ satisfies the following properties:

(i) $\operatorname{ent}(H)=0$ if and only if $H$ is a crisp set.

(ii) $\operatorname{ent}(H)=1$ if and only if $H \in Q(X), T_{H}^{+}(x)=C_{H}^{+}(x)=U_{H}^{+}(x)=$ $F_{H}^{+}(x)=T_{H}^{-}(x)=C_{H}^{-}(x)=U_{H}^{-}(x)=F_{H}^{-}(x)=0.5 \forall x_{i} \in X$.

(iii) $\operatorname{ent}(H) \geq \operatorname{ent}(K)$ if and only if $T_{H}^{+}\left(x_{i}\right)+F_{H}^{+}\left(x_{i}\right) \leq T_{K}^{+}\left(x_{i}\right)+F_{K}^{+}\left(x_{i}\right), T_{H}^{-}\left(x_{i}\right)+$ $F_{H}^{-}\left(x_{i}\right) \geq T_{K}^{-}\left(x_{i}\right)+F_{K}^{-}\left(x_{i}\right),\left|C_{H}^{+}\left(x_{i}\right)-U_{H}^{+}\left(x_{i}\right)\right| \leq\left|C_{K}^{+}\left(x_{i}\right)-U_{K}^{+}\left(x_{i}\right)\right|, \mid C_{H}^{-}\left(x_{i}\right)-$ $U_{H}^{-}\left(x_{i}\right)|\geq| C_{K}^{-}\left(x_{i}\right)-U_{K}^{-}\left(x_{i}\right) \mid$.

(iv) $\operatorname{ent}(H)=\operatorname{ent}\left(H^{c}\right)$.

Proposition 4.14. One can easily verify that ent $(H)=1-\frac{1}{4 n} \sum_{i=1}^{n}\left[\left(T_{H}^{+}\left(x_{i}\right)+\right.\right.$ $\left.\left.F_{H}^{+}\left(x_{i}\right)\right)\left|1-\left(U_{H}^{+}\left(x_{i}\right)+C_{H}^{+}\left(x_{i}\right)\right)\right|+\left\{\left(U_{H}^{-}\left(x_{i}\right)+C_{H}^{-}\left(x_{i}\right)\right)\left|1-\left(T_{H}^{-}\left(x_{i}\right)+F_{H}^{-}\left(x_{i}\right)\right)\right|\right\}\right] \forall x_{i} \in$ $X$ is an entropy measure of a BQSVNS $H \in Q(X)$.

Remark 4.15. One can easily show that ent $(H)$ is a possible similarity measure of the BQSVNS $H$. However we have calculated the entropy measure of the BQSVNS $A$ given in Example 3.2. We have seen that $\operatorname{ent}(A)=\operatorname{ent}\left(A^{c}\right)=0.89312$.

\section{A decision making problem}

A medical diagnosis problem can very well be represented by using a BQSVNS model. Similarity measure between two BQSVNS is the most 
powerful technique to solve real life medical problems. Using these similarity measure technique, we can easily say whether a patient is effected by a particular disease or not. In rainy season, Dengue disease spreads rapidly in Kolkata sub-urban area of West Bengal, India. The patient of these disease has primarily 4 symptoms, say headache, high fever, cough, rashes in the body. But sometimes these symptoms are not clearly seen sometime in every dengue patient. Also these symptoms are common in many different viral infections. As a result, every patient must undergo some blood tests in a city clinic for confirmation of dengue. Thus for a doctor who lives in a rural area without proper infrastructure, have to diagnosis a patient with dengue fever on intuition basis. Here we have developed a BQSVNS based method for the detection of dengue fever in patients using similarity measure technique.

We have tried to develop a BQSVNS based model of these particular problem for detecting a patient suffering a dengue patient or not and tried to solve it by similarity measure technique.

Suppose $x_{1}, x_{2}, x_{3}, x_{4}$ denote the symptoms headache, high fever, cough, rashes in the body respectively. We have considered three patient say $C_{1}, C_{2}, C_{3}$ out of which $C_{1}$ is a confirmed dengue patient. $C_{1}$ will be considered as a model case for dengue disease. On the other hand $C_{2}, C_{3}$ have dengue symptoms but both of them did not undergo any blood test for confirmation. We have represented the condition of these patient as a BQSVNS as follows:

$$
\begin{aligned}
& C_{1}=\langle 0.8,0.5,0.5,0.5,-0.1,-0.2,-0.2,-0.1\rangle / x_{1}+ \\
&\langle 0.6,0.4,0.5,0.6,-0.5,-0.3,-0.2,-0.1\rangle / x_{2}+ \\
&\langle 0.4,0.5,0.6,0.4,-0.2,-0.1,-0.1,-0.2\rangle / x_{3} \\
&+\langle 0.6,0.5,0.6,0.6,-0.1,0,0,0\rangle / x_{4} . \\
& C_{2}=\langle 0.9,0.6,0.7,0.2,-0.5,-0.4,-0.1,-0.8\rangle / x_{1}+ \\
&\langle 0.5,0.8,0.60 .4,-0.5,-0.4,-0.6,-0.5\rangle / x_{2}+ \\
&\langle 0.4,0.6,0.7,0.8,-0.6,-0.5,-0.6,-0.4\rangle / x_{3} \\
&+\langle 0.6,0.5,0.8,0.6,-0.4,-0.8,-0.6,-0.5\rangle / x_{4} . \\
& C_{3}=\langle 0.9,0.5,0.5,0.6,-0.5,-0.2,-0.4,-0.3\rangle / x_{1}+ \\
&\langle 0.6,0.8,0.6,0.4,-0.5,-0.5,-0.4,-0.4\rangle / x_{2}+ \\
&\langle 0.6,0.5,0.6,0.8,-0.6,-0.4,-0.8,-0.4\rangle / x_{3} \\
&+\langle 0.4,0.6,0.7,0.9,-0.4,-0.6,-0.7,-0.5\rangle / x_{4} .
\end{aligned}
$$


In order to determine whether $C_{2}, C_{3}$ has dengue disease or not, we now calculate the similarity measure of them with $C_{1}$. We will consider that a patient is suffering from dengue if its similarity with $C_{1}$ is about 0.35 . After calculating this, we have the following result:

$$
\begin{aligned}
S^{d}\left(C_{1}, C_{2}\right) & =0.10753, S^{d}\left(C_{1}, C_{3}\right)=0.11905 \\
S^{\prime}\left(C_{1}, C_{2}\right) & =0.31882, S^{\prime}\left(C_{1}, C_{3}\right)=0.35253 \\
S^{c}\left(C_{1}, C_{2}\right) & =0.33169, S^{c}\left(C_{1}, C_{3}\right)=0.35218 .
\end{aligned}
$$

Based on the above results, it is clearly seen that $C_{3}$ 's condition is much more similar to $C_{1}$ 's condition than $C_{2}$ 's condition. Hence, the chance of having dengue disease of $C_{3}$ is much higher than $C_{2}$.

\section{Conclusion}

In this paper, we have developed the concept of BQSVNS which is an extension of quadripartitioned single valued neutrosophic set and a bipolar set. We also discuss different types of terminology of BQSVNS and develops set theoretic operations on it. Beside that, we have defined different types of similarity measures with examples. Finally we have solved a decision making problem based on bipolar quadripartitioned single valued neutrosophic set. In real world, there are many such examples exist where four valued logic works. In 1977, Prof N.D. Belnap showed the modern uses of multiple valued logic in his paper [3]. But it is quite natural that for each logic there is a positive membership degree as well as negative membership degree. For this reason, bipolar sets are developed. Bipolar concept together with four valued logic develops the idea of BQSVNS. It is a generalization among a all the previous sets. In future, we will apply our newly developed set as a model to real world problems with realistic data and extend it to other decision making problems. Also one may try to extend it to tripolar or multipolar quadripartitioned single valued neutrosophic sets. 


\section{References}

[1] K. Atanassov, "Intuitionistic fuzzy sets", Fuzzy sets and systems, vol. 20, no. 1, pp. 87-96, Aug. 1986, doi: 10.1016/ S0165-0114(86)80034-3

[2] A. Aydoğdu, "On similarity and entropy of single valued neutrosophic sets", General mathematics notes, vol. 29, no. 1, pp. 67-74, Jul. 2015. [On line]. Available: https:/ / bit.ly/ 3oW0yrm

[3] N. D. Belnap, "A useful four-valued logic", in Modern uses of multiplevalued logic, J. M. Dunn and G. Epstein, Eds. Dordrecht: Springer, 1977, pp. 5-37, doi: 10.1007/978-94-010-1161-7_2

[4] S. Broumi and F. Smarandache, "New distance and similarity measures of interval neutrosophic sets", 17th International Conference on Information Fusion (FUSION), Salamanca, 2014, pp. 1-7. [On line]. Available: https:/ / bit.ly/ 2TWb9nP

[5] R. Chatterjee, P. Majumdar, and S. K. Samanta, "On some similarity measures and entropy on quadripartitioned single valued neutrosophic sets", Journal of intelligent and fuzzy systems, vol. 30, no. 4, pp. 2475-2485, 2016, doi: 10.3233/ IFS-152017

[6] I. Deli, M. Ali, and F. Smarandache, "Bipolar neutrosophic sets and their application based on multi-criteria decision making problems", 2015 International Conference on Advanced Mechatronic Systems (ICAMechS), Beijing, 2015, pp. 249-254, doi: 10.1109/ ICAMechS.2015.7287068

[7] F. Karaaslan, "Neutrosophic soft sets with applications in decision making", International journal of information science and intelligent system, vol. 4, no. 2, pp. 1-20, 2015, doi: 10.5281/ zenodo.23151

[8] K. Lee, Bipolar-valued fuzzy sets and their operations, Proceedings of the International Conference on Intelligent Technologies, Bangkok, Thailand, 2000, pp. 307-312, 2000

[9] K. Lee, "Bipolar fuzzy subalgebras and bipolar fuzzy ideals of BCK/ BCI algebras", Bulletin of the Malaysian Mathematical Sciences Society, vol. 32, no. 3, pp. 361-373, 2009. [On line]. Available: https:/ / bit.ly/ 35Y4lvu

[10] P. Majumdar, "Neutrosophic sets and its applications to decision making", in Computational intelligence for big data analysis, D. P. Acharjya, S. Dehuri, and S. Sanyal, Eds. Cham: Springer, pp. 97-115, 2015, doi: 10.1007/978-3-319-16598-1_4 
[11] J. M. Merigo and M. Casanovas, "Decision making with distance measures and induced aggregation operators", Computers and industrial engineering, vol. 60, no. 1, pp. 66-76, Feb. 2011, doi: 10.1016/j.cie.2010.09.017

[12] J. M. Merigo and M. Casanovas, "Induced aggregation operators in the Euclidean distance and its application in financial decision making", Expert systems with applications, vol. 38, no. 6, pp. 7603-7608, Jun. 2011, doi: 10.1016/j.eswa.2010.12.103

[13] J. M. Merigo and R. R. Yager, "Generalized moving averages, distance measures and OWA operators", International journal of uncertainty, fuzziness and knowledge-based systems, vol. 21, no. 4, pp. 533-559, 2013, doi: 10.1142/ S0218488513500268

[14] A. B. Saeid, "Bipolar-valued fuzzy BCK/ BCI-algebras", World applied sciences journal, vol. 7, no. 11, pp. 1404-1411, 2009. [On line]. Available: https:/ / bit.ly/ 310cUfR

[15] V. Santhi and G. Shyamala, Notes on bipolar-valued multi fuzzy subgroups of a group, International journal mathematics archive, vol. 6 , no. 6, pp. 22295046, 2015. [On line], Available: https:/ / bit.ly/ 3erUn9x

[16] F. Smarandache, "Neutrosophic set-a generalization of the intuitionistic fuzzy set", 2006 IEEE International Conference on Granular Computing, Atlanta, GA, USA, 2006, pp. 38-42, doi: 10.1109/ GRC.2006.1635754

[17] F. Smarandache, "A geometric interpretation of the neutrosophic set - A generalization of the intuitionistic fuzzy set", 2011 IEEE International Conference on Granular Computing, Kaohsiung, 2011, pp. 602-606, doi: 10.1109/ GRC.2011.6122665

[18] F. Smarandache, "Neutrosophic Set - a unifying field in set", in A unifying field in logics : neutrosophic logic : neutrosophy, neutrosophic set, neutrosophic probability, 2nd ed., Rehoboth, DE: American Research Press, 2000, pp. 111-114. [On line]. Available: https:/ / bit.ly/ 3oVpzTz

[19] H. Wang, F. Smarandache, Y. Zhang, and R. Sunderraman, "Single valued neutrosophic sets", Review of the Air Force Academy, no. 1, pp. 10-14, 2010. [On line]. Available: https:/ / bit.ly/ 3oWGd5l

[20] J. Ye, "Vector similarity measures of simplified neutrosophic sets and their application in multicriteria decision making", International journal fuzzy systems, vol. 16, no. 2, pp. 204215, 2014. [On line]. Available: https:// bit.ly/ 38pL7SH 
[21] J. Ye and Q. Zhang, "Single valued neutrosophic similarity measures for multiple attribute decision-making", Neutrosophic sets and systems, vol. 2, pp. 48-54, Sep. 2020. [On line]. Available: https:/ / bit.ly/ 387ppTc

[22] J. Ye and Q. Zheng, "Dice similarity measure between single valued neutrosophic multisets and its application in medical diagnosis", Neutrosophic sets and systems, vol. 6, pp. 48-52, 2014, doi: $10.5281 /$ zenodo. 22448

[23] J. Ye and Q. Zheng, "Multi-period medical diagnosis method using a single valued neutrosophic similarity measure based on tangent function", Computer methods and programs in biomedicine, vol. 123, pp. 142-149, Jan. 2016, doi: 10.1016/j.cmpb.2015.10.002

[24] L. A. Zadeh, "Fuzzy sets", Information and control, vol. 8, no. 3, pp. 338-353, Jun. 1965, doi: 10.1016/ S0019-9958(65)90241-X 